\title{
Towards the Feasibility of Instituting a Philippine Digital Audio Library: A Case Study
}

\author{
Jia Li \\ Kolej International College, Krirk University, Maha Nakhon 10220, Thailand; 1146043158@qq.com
}

To cite this article (APA): Li, J (2021). Towards the Feasibility of Instituting a Philippine Digital Audio Library: A Case Study. Journal of ICT in Education, 8(2), 1-12. https://doi.org/10.37134/jictie.vol8.2.1.2021

To link to this article: https://doi.org/10.37134/jictie.vol8.2.1.2021

\begin{abstract}
As a spearhead force in music research, especially in the area of South East Asia region, the University of the Philippines (UP) Center for Ethnomusicology (UPCE) caters to a gigantic collection of audio materials which covers different musics and musical traditions in the Philippines, South East Asia and representative areas from other continents. As an outcome of its former appellation, the "UP Ethnomusicology Archives", UPCE hosts an ethnomusicological collection of about 2500 hours of recorded music in open reel and cassette tape formats, under the authorship of Jose Maceda whose visionary work of putting together these valuable recorded materials left a treasure for ethnomusicology scholarship and research. In recognition of his influential contribution that made the UCPE an archive and repository of materials on music, philosophy, anthropology and other cognate disciplines, these audio materials, together with field notes, music transcriptions, song texts, photographs, music instruments, music compositions, personal files, about 200 books and journals, all of which he personally initiated and developed as a unified institution resource for music research are called "Jose Maceda Collection".
\end{abstract}

Keywords: digital audio library, ethnography, ethnomusicology.

\section{INTRODUCTION}

As an important way of expression of human life and feelings, music has a history as old as human being itself. As a common language which can be shared across the boundary of cultures and geography, music stores and preserves ideas and norms that can establish a unique cultural identity of people. So technological development comes along the needs of recording, storing, transforming and distributing the sound material and makes it passed on from generation to generation, and forging a narrative of the people's voice, history and life. The field of libraries and research centers has been impacted greatly since the age of digitization came and the researchers nowadays carry on the 
responsibility to inherit and update the cultural heritage. Ethnographers have actively tailored rather than passively transposed ethnography to the study of expertise. Departing from traditional conceptions of ethnography, these works exhibit growing attentiveness to movement, mediation, and materials. We argue that this retooling of ethnography is not merely a response to empirical realities but rather stems, at least in part, from the influence of science and technology studies, specifically Actor-Network Theory (Graizbord et al., 2017).

Digitization as a preservation strategy has been the subject of debate among the members of the cultural heritage community for two decades. The benefits of digitization in expanding access are universally acknowledged, but the recognition of digitization as an option for long-term preservation of analogue materials is still controversial (Matusiak et al., 2012). The term, 'digitalization' involves the process of converting information, such as texts, images, video, and audio, into a digital format that can be stored in, accessed, and transmitted through electronic devices. The new possibilities, that the digitalization brought with it, has changed how music is produced, distributed, and consumed. In this way, the digitalization has transformed the music industry (Muller, 2013).

Amongst several advantages of digitization, some of the most noteworthy are:

- Space required for storage of material is saved;

- Easy and faster access to any type of material;

- Barriers of space and time in accessing the information are overcome;

- Creation of multiple copies has become easier; and

- Preservation for posterity is facilitated.

The digital revolution is giving oral historians exciting new ways to record, index, search, and share oral history interviews with larger and more remote audiences. Foe example, the Illinois State Museum's Oral History of Illinois Agriculture (OHIA) project used an array of digital methods and tools to develop an interactive website, called the Audio-Video Born, which gives voice to people involved in agriculture and rural life in Illinois. The OHIA approach can serve as a model for anyone looking for engaging new ways to share oral histories with community audiences. First, the AudioVideo Born joins a growing chorus of websites that go beyond the limitations of printed words in traditional oral history transcripts; it gives visitors access to primary-source audio and video recordings that restore emotion and meaning to the stories being told. Second, the Audio-Video Born uses digital indexing of audio and video recordings to make them searchable in a database format. Finally, the Audio-Video Barn opens its doors to provide free access to searchable recordings via the Internet, making them widely available to diverse audiences (Warren et al., 2013). In the fired of music, technology has also fundamentally transformed the way researchers and the public store and access to the music materials, which are a narrative of the history of a group of people or even a nation. Traditionally, audio collectors focused on formats from across the twentieth century, including wax cylinders, 78s, LPs, and CDs. In the more recent times, internet access including cataloging, streaming and downloading calls for projects to more easily store and open access the data to the public use (Justice, 2015). In this case, digitization is the first step towards the establishment of such an audio 
library. Smith (2015) presented his observations of what it means to identify as an audio collector in an era where abundance replaces scarcity and streaming access substitutes for physical formats such as access, sharing, and participating become more important; music functions as service more than products (Justice, 2013).

\section{JOSE MACEDA COLLECTION DIGITIZATION PROJECT}

The Jose Maceda Collection Digitization Project (UJMCDP) is a pioneering endeavor at UPCE to digitize the audio recordings, field notes and photo of the whole collection with modern digital technology, which helps prolong the limited life span of previous venerable medias to unlimited access and time. The primary goal of this digitation project is to preserve and provide open access for scholars and performing artists to these valuable first-hand resources. In this paper, I will focus on the digitization of audio materials, which were recorded on the medias of analogue formats such as cassette tapes and open reels. I will explain in details the systematic, swift and economic process that the digitization working team has been following all the way long. Visual examples of the process will be provided to highlight the critical facilities and techniques. The objective of this paper is to establish a model in the Philippines to digitize institution-wise analogue audio materials which can be shared by open public and build up a similar digital audio library.

The core of the entire audio collection represents the traditional music cultures of 78 major ethno linguistic groups across the Philippine archipelago as well as field recordings of selected music cultures in South East Asia, including Indonesia, South china and Malaysia. To digitize this collection from analogue formats will serve four major purposes:

1. To make restorative and preventive conservation;

2. To establish a new catalogue and systematic musicological database;

3. To provide access towards a digital audio library; and

4. To provide a title/song-based song indexing/search engine.

This project is currently being fonded by the National Commission for Culture and Arts with supporting grants from the chancellor of the University of the Philippines, Diliman and the Jikji Prize of the Austrian Academy of Science. On the technique aspect, the project team received considerable help from Phonogrammarchiv, an Austria based archive center, which provided human resource training and technical assistance.

Starting from November, 2009, the project team have almost finished the entire process of digitization of 1530 open reels of Filipino music and around 800 open reels of international sources, as well as 191 cassette tapes, which equates to around 2500 running hours of music. The current catalogue up to August 2012 has already accumulated 2649 titles, with the steadfast expansion as the project is still going on. 
The whole processes were carried by the UPCE sound laboratory whose manpower consist of college achivist David Dino Guadalupe and his two student assistants. The whole project has been under the direct leadership of UPCE Executive Director, Emeritus Professor Dr. Ramon Santos and UPCE Advisory Board. Figure 1 shows the UPCE Sound Laboratory.

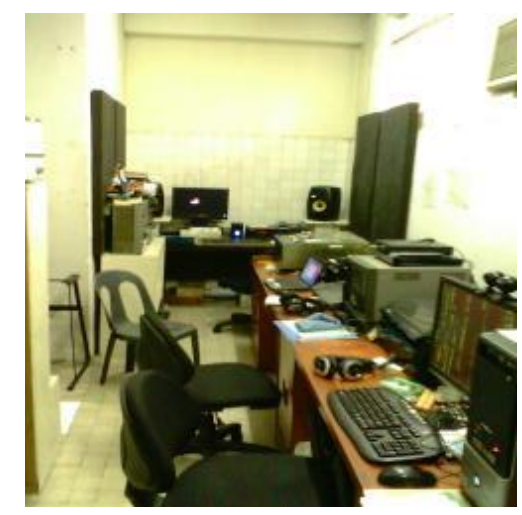

Figure 1: The UPCE Sound Laboratory

\section{AUDIO MATERIAL DIGITALIZATION}

In the area of library science, digital music library project has been arousing more and more interest from the academies in the past decade. In 2000, Amanda Maple and Tona Henderson described the issues that must be confronted by a librarian planning a digital music library project, and explained the decisions made for their own project at Pennsylvania State University. The issues fall into three broad areas which are infrastructure (including the selection of hardware, software, streaming technology, and method of access); collections (including decisions on what to digitize and why, and related questions of copyright); and staffing (including who does what, who employs them, how the work is funded, and who provides training and public service) (Griscom, 2003).

A music recording provides a mean to unlimited access to a historical music event through electronic or mechanical inscription and re-creation of sound waves. The main two categories of recording technology are analogue recording and digital recording. Though historically speaking, analogue recording enjoys much merits in music events capture, due to its shortcomings such as distortions, speed variations, noise/hiss, low accessibility and deterioration of media with the passage of time, digitization of such material seems to be extremely necessary for both achieval and scholarly purpose.

Digitization is the process of transforming analogue materials into its digital formats which can be more easily accesses for use and help preserve the recorded material, allowing it to be stored and transmitted by a wider variety of medias. From a more technical term, digitization is the process of converting information - analogue audio, text, and/or visual images - into a digital format by 
organizing information into units of data called bits that could be processed by computer related equipments. Instead of dealing with easily decayed and fragile original materials, digitization provides with extraordinary access and availability of information, as well as serving for the pedagogical purposes through computer network locally or internationally. The whole working process and flow can be graphed as in Figure 2 which consist of five steps as content digitization, content storage, content editing, content cataloguing, and content distribution.

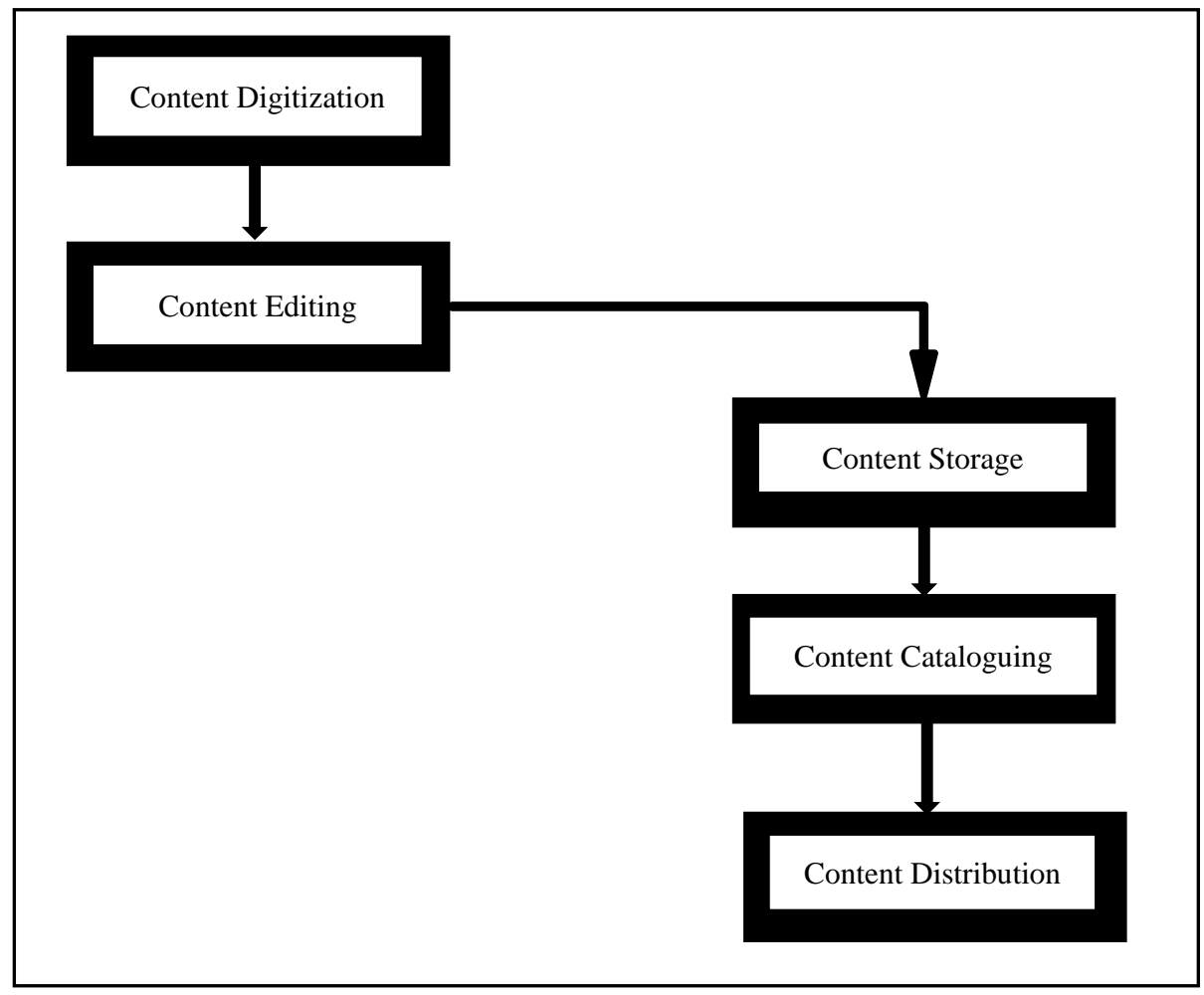

Figure 2: Audio Digitization Working Flow

\section{Step 1: Content Digitization}

The digitization audio workstation has the capability as follows: CPU dual core 2.4GHZ, DDR2 RAM 2GB, two hard disk drives (80GB and 150GB), with the operating system installed as windows XP. The audio interface is RME fireface 400. For the signal input equipment, a Studer A807 MKII function as open reel player and Tascam CD-A750 as cassette tape playback deck. The major software in the digitization is Wavelab 6 by Steinberg, together with two signal and spectrum analyzing softwares, digicheck (bundled tree from the RME interface) and Scan Input. The Studer and Tascam CD used are shown in Figure 3. 

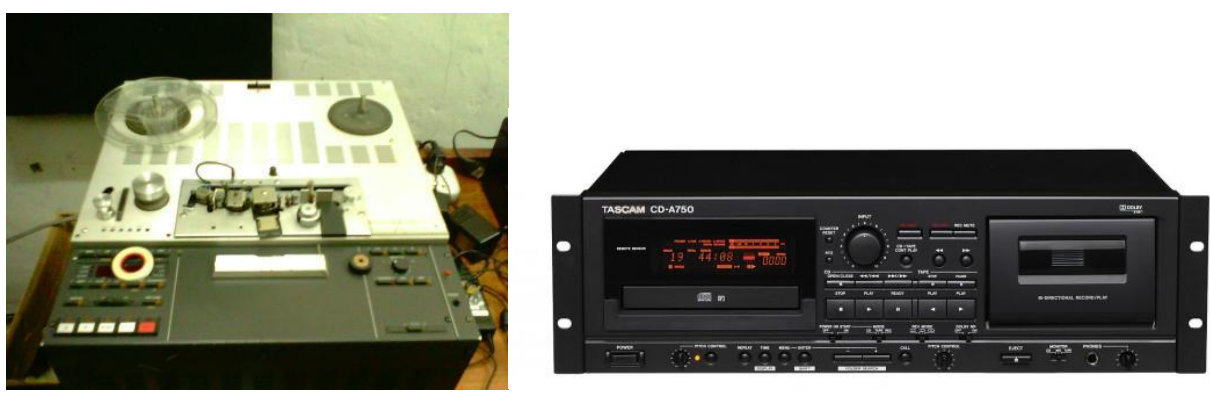

Figure 3: Studer A807 MKI Open Real Recorder (left); Tascam CD-A750 (right)

Due to the critical conditions of the old analogue open reels, some restoration may be required to be done before the actual digitization. Some open reels need to be extended with lead tape at the two ends by 15 inches. Some need to be replaced of the old splicing tapes (as shown in Figure 4) which were extremely fragile already. A good advantage of Studer A807 MKII is that it provides with a digital counter which can pin down and locate precisely any point of the music as you need, to avoid unnecessary rewiring and break the tape. The ready-to-convert open-reel were delivered to the Sound Laboratory at least 24 hours before the digitization to assure the tapes to be adjusted to the humidity and other environmental factors of the laboratory.

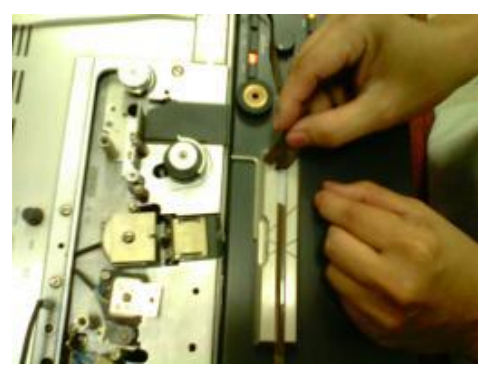

Figure 4: Slicing Tape Replacement

The different speeds that the music was recorded produced different specifications of frequency response at record/reproduce mode. Table 1 shows the most frequently encountered speed ratio during this project and their corresponding frequency response. For different types of open reel and corresponding total play time, Table 2 shows the approximating playing times for different reel sizes (one side).

Table 1: Analogue Recoding Speed Ration and Frequency Response

\begin{tabular}{lllll}
\hline \multicolumn{1}{c}{ Speed ratio } & & 3.75 ips & \multicolumn{1}{c}{ 7.5ips } & 15 ips \\
\hline Frequency & $+/ \_2 \mathrm{~dB}$ & $30 \mathrm{HZ} \sim 12 \mathrm{KHZ}$ & $30 \mathrm{HZ} \sim 16 \mathrm{KHZ}$ & $30 \mathrm{HZ} \sim 20 \mathrm{KHZ}$ \\
Response & $+/ \_1 \mathrm{~dB}$ & $30 \mathrm{HZ} \sim 8 \mathrm{KHZ}$ & $30 \mathrm{HZ} \sim 12 \mathrm{KHZ}$ & $30 \mathrm{HZ} \sim 18 \mathrm{KHZ}$ \\
\hline
\end{tabular}


Table 2: Different Types of Open Reel and Approximating Playing Time (Open Side)

\begin{tabular}{lccc}
\hline $\begin{array}{c}\text { Type of Open Reel } / \\
\text { Recording Speed }\end{array}$ & 5” Reel 600ft & 7" Reel 1200ft & 10.5” Reel 2500ft \\
\hline $17 / 8$ ips & 1 hour & 2 hours & 4 hours \\
3 3/4 ips & 30 minutes & 1 hour & 2 hours \\
$71 / 2$ ips & 15 minutes & 30 minutes & 1 hour \\
15 ips & - & - & 30 minutes \\
\hline
\end{tabular}

Before digitization recording into workstation, the project team would use Digicheck to analyze the signal peak and frequency response range. If these factors showed symptoms of distortion or other undesirable situations, the playback head of the open reel player or cassette tape player would be adjusted accordingly. A good alignment of input signal level is of vital importance, especially in the case of the defective recorder during the original recording session. Digicheck's signal graphics engine, as shown in Figure 5 (left) would analyze and monitor a real time signal level match, which would be reflected as the expansion of both low and high frequency. Especially the high frequency part is the more important indicator of an input recording level. Furthermore, Scan Input Spectrum Analyzer, as shown in Figure 5 (middle) can be applied to minimize the noise level of the recording, the signal spectrum showcase the real time noise/signal ratio as reflected in the color change.
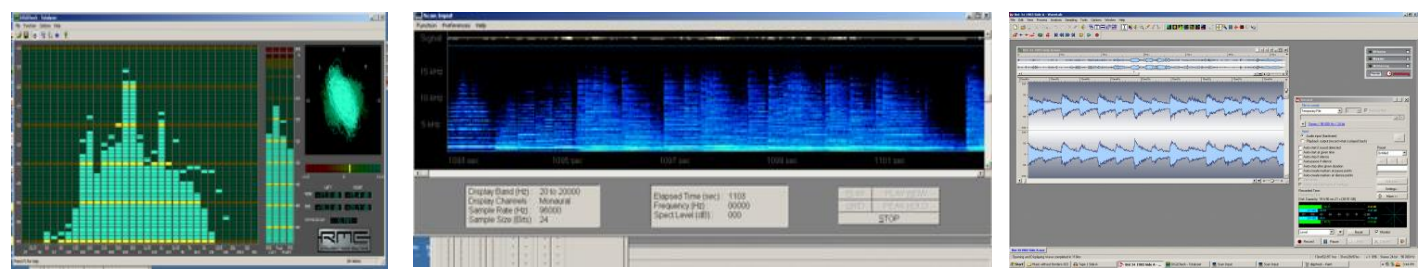

Figure 5: Diticheck Signal Analyzing Interface (left); Scan Input Spectrum Analyzer (middle); Wavelab 6 Main Interface (right).

After these initial stages, the digitization recording would be starting, the Steinberg Wavelab 6, as shown in Figure 5 (right) was used as digitization producer, which provided a full range of possibility in saving formats. At this stage, the files were saved into two formats which are wav $(96 \mathrm{kHZ} / 24 \mathrm{Bit})$ and mp3. The former is for archival purpose, while the later would be for further editing. The different digital saving formats during this project will be discussed in the section of content storage.

\section{Step 2: Content Editing}

Due to the nature of analogue recording of open reels and cassettes, one side of open reel provides a variety length of playing time, as previous shown in Table 2, depending on the length of the tape and recording speed ratio. The same case is with cassette tapes. Each side consists of a various numbers of songs by the same or different researchers. To serve for the purpose of easily search, indexing, and 
appreciation purpose, the next step was to edit the audio file and cut it by the unit of song/title with corresponding metadata.

This stage was completed at a separates editing workstation, transfer by external hard disk saved in mp3 format. The specifications of the editing workstation are: dual core $3.0 \mathrm{GHz}$ CPU, 2GB DDRII memory with Linux/windows XP dual operating system. The audio editing software was audacity 1.312 - beta, which is a free software for downloading. The advantage of the software is that it operates on a copy of the original file instead of physical changing realtime, which provides high data security.

There are two main issues need to be on mind during this stage. Firstly, some audio file need to change speed to its reel tempo. In early readings, some were conducted at an extremely low tempo. For example, the open reel with old catalogue number of Bikol-RK-1-1967 was a performance recorded on ST816 machine live in 1967 by Jose Maceda in Bikol. The side A was recorded at speed of 17/8 ips while side B at $33 / 4$. However, this extremely low tempo at side A is beyond the capacity of the equipment available in the sound laboratory which could only reach as low as 3 ips. This means the current digitized archival data is actually much faster than the real performance. At the editing stage, the project team need to adjust the music speed back to tempo digitally. This can be achieved by using the speed change function which locates at "Effect - change speed", as shown in Figure 6 (left).
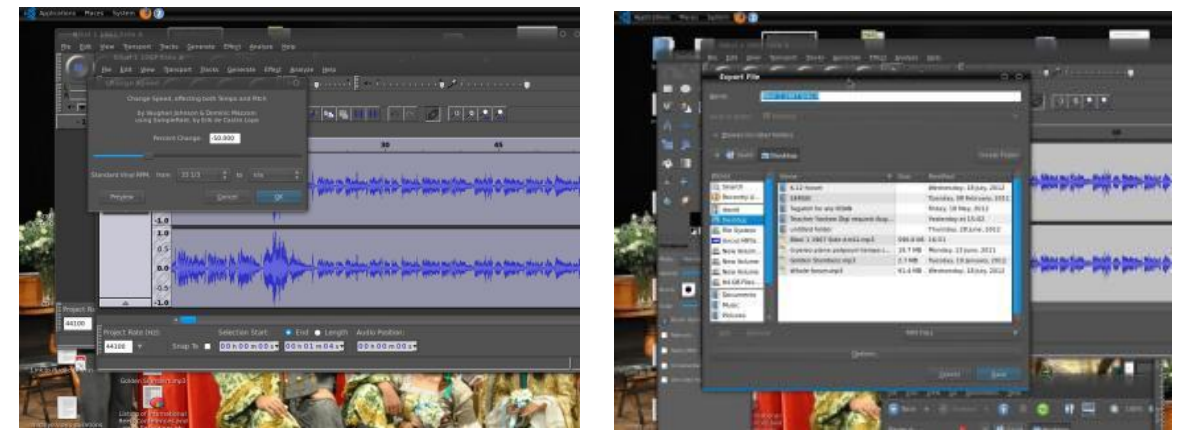

Figure 6: Audacity's Speed Change Window (left); Audacity’s File Export Window (right).

Secondly the context cutting and slicing demands a high expertise in music understanding, to assure the cutting point is at the right place. This professionalism differentiates the digitization of audio materials towards a digital audio library from other casual digitization projects. On top of the fact that all the members had the processional training and background in music research, the project team actually listened to all the music, corresponding to the original data from the recordings, to locate exactly where the song stopped, rather than mechanically relying on wave spectrum which is unsecure.

After done all these to secure the accurate starting and ending point of each title, the selection of audio data, which was in the original speed and song by unit, was saved again in $\mathrm{mp} 3$ and ogg formats to two new folders, as shown in Figure 6 (right), by using "file - export selection". The reason that saving in these two formats will be explained thereafter. The metadata from the original source was tagged, including song title, author, year, genre, and other necessary information. 


\section{Step 3: Content Storage}

The external storage device was 6 sets of Western Digital mirror edition Caviar ${ }^{\mathrm{R}}$ Green $^{\mathrm{TM}}$. The advantage of this external hard disk drive is its automatic back up function which would be pertinent for archival setting. One set of hard disk consist two units, which function as a mirror to each other. Besides, it provides upgradability for capacity expansion in the future. Its reliability as shown in its cool and quiet operation guarantee its longer playing time which is valuable for such digitization of mega data process that usually takes hours. Its USB connection to the computer provides easy and flexible data storage and transfer.

The project team identified three types of usage/purposes for the digitized data, namely archival, academic, and distribution. Three types of formats were used to address these different needs, as reflected in three versions in the content storage during the whole process, which are archival version, research version and web version, taking into consideration of such factors as data size, technical specifications, and others.

The three version of digital audio data were stored in separate sets of hard disk. The archival version would be at UPCE archival storage. The researcher version would be forwarded to UPCE library for any scholarly research, which can be easily uploaded to a computer or digital CD or DVD. The online version would be for website uploading which would be still a process to follow in the future.

1. Archival version. The digitalized original files were saved in $96 \mathrm{KHZ} / 24$ bit wave format audio file format for archival purpose. These are all unedited uncompressed data from the initial digitizing. So the file is saved by per tape side. Wav audio format is a Microsoft and IBM audio file format standard for storing an audio bits' stream on PCs. It is the main format for raw and typically uncompressed audio.96HZ/24Bit specification provides a hi-fidelity of listening and space for future digital sound encoding or publication purpose while in the meantime does not make the size overwhelming.

2. The research version from the digital editing was saved in MPE G-2 Audio Layer III (MP3) format. The file is saved by title/song unit with new accession number to be allocated in the next stage. Mp3 format is a patented encoding format for digital audio which uses a form of lossy data compression. However, the compression was operated by reducing accuracy of certain parts of sound. This are considered to be beyond the auditory resolution ability of most people. Due to its much smaller size comparing to wave file, it is the ideal format for easily uploading to any computer at UPCE library for any listening and academic purposes.

3. The web version from the digital editing was saved in ogg format. These data are the same as in the edited compressed research version, but even smaller. The ogg digital format is format is a free, open container format maintained by the Xiph. org Foundation. Because the format is free, and its reference implementation is not subject to restrictions associated with copyright. Industry wise, its rather small size and high compatibility with different free and proprietary media players as well as portable media players, make it an ideal choice for web-based content distribution. 


\section{Step 4: Content Cataloguing}

A systematic approach towards cataloguing is of vital importance for instant locate a specific title/tune digitally as well as physically. Up to August 25, 1012, the Jose Maceda Digitization Project has already contributed 2649 songs/ titles to UPCE digital audio library. How to deal with such big number of audio data, based on the old paper book catalogue of the original sources (open reels, cassette tapes), a new digital catalogue was established for both library indexing and web search date base purpose.

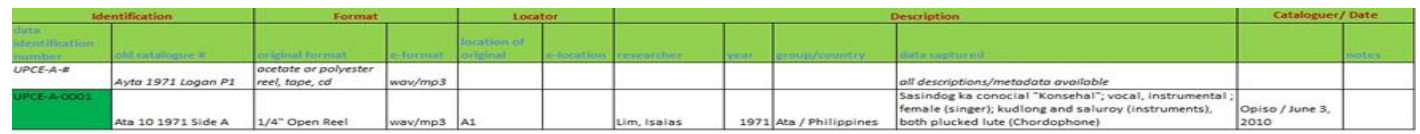

Figure 7: UPCE Digital Audio Catalogue

As shown in Figure 7, the new digital catalogue consists of such parameters as: data identification number (title accession number), old catalogue number, original format, digital format, location of the original data, electronic location, researcher, year, ethnic group, country of origin, metadata description, and others. This provided an efficient system to instant search online or at UPCE library by providing key words.

\section{Step 5: Content Distribution}

To invite scholars, artists, pedagogues and students to make maximum use of such gigantic digital audio database, join and interact with CDCE in exploring traditions in the in the musical world, three ways of accession were provided.
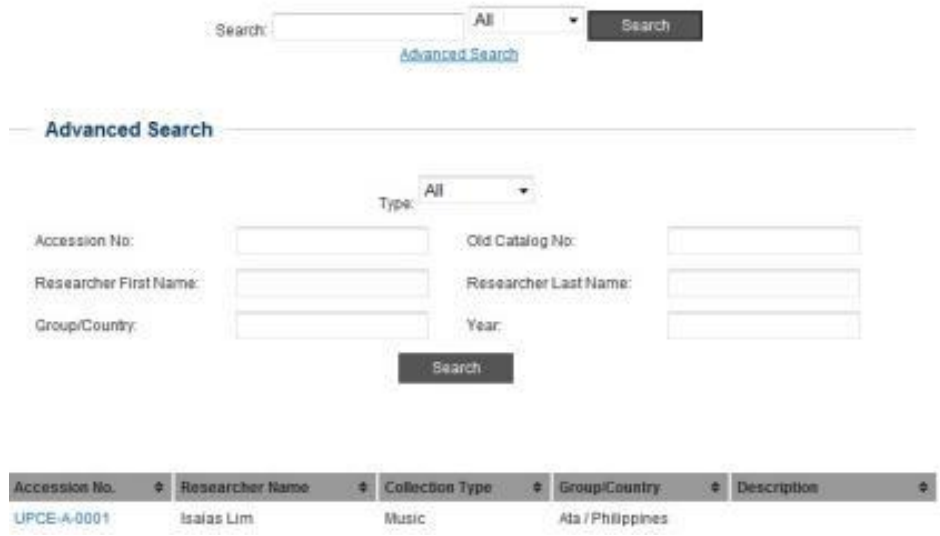

Figure 8: Search Engine of UPCE Jose Maceda Digital Audio Library 
Firstly, a website hosted at UPCE website (www.upcenom.com) was exclusively created for this digital audio collection. A powerful search engine (as shown in Figure 8) was design for instant access by accession number, researcher, ethnical group, country of origin or year of recording. Secondly, this collection is open to public for on-site listening that audio data will be uploaded to the computer at UPCE library by simply filling a request form. Lastly, for special requests, UPCE will be able to save the requested data on (CD or DVD) and mail to requested locations locally or internationally.

\section{REFLECTION ON THE PROJECT}

This paper showcased a cost-effective approach towards an institution - wise digitalize audio library establishment, which can be easily apply to other institutions that facing the same problems and situations with even very limited funding and human resource. However, there are two important areas of concern that I would like to recommend for further enhancing the scope of digital audio library projects.

Firstly, this initial project on digitizing Jose Maceda Collection aims to preserve the original analogue material which demands authenticity of digitized copy as to the original sources. However, with modern digital audio technology, it is not difficult to enhance the current audio signal, such as noise elimination, post-production effect, etc. This extension of the digitization audio projects can further produce audio data at the fidelity ratio sufficient for publication in CD or DVD formats which can reach a wider audience of households.

Secondly, the Jose Maceda collection also consist vast amount of related ethnomusicological materials, like musical transcriptions, transcribed texts, photos, field notes, etc. How to incorporate these data as meta data into the digital audio library is an important topic to solve to boost up the serviceability of the current digital audio library to a higher level in the future.

Lastly, how to establish a more efficient music retrieval system which can describe the current audio data with more musicological data will be of vital importance for future scholarly research. New advancements in digitization like music information retrieval system, Melody Indexing System (MELDEX), adaptive content-based music retrieval system have already paved way for this purpose, from a technological perspective in general. However, how to install an efficient system which serves best the music of indigenous culture and record at old analogue technology live with low-fidelity is still calling for the collaboration and endeavor among musicologist, library scientists and engineering scientists. In this perspective, the establishing of digital audio library is not a simplistic technical issue, but rather an integral part of ethnomusicological research that has been providing new knowledge and data to support and enhance this area of scholarship. 


\section{CONCLUSION}

Digitization is an ever-evolving process which cumulate along side with the advancement of digital information technology. In this sense, the currently on going Jose Maceda Digital Audio Library Project is not an end in its own term, but rather open a new door towards a national and international - wise digital audio library with a mutual aim towards enchancing scholarly research, share musicological database and promote different indigenous or regional musical traditions and cultural identities.

\section{ACKNOWLEDGEMENT}

The first draft of this paper was presented at the 5th International Conference with the theme, Libraries, Archives and Museums: Common Challenges, Unique Approaches, October 25-26, 2012 at the Leong Hall Auditorium, Ateneo de Manila University, Quezon City, Philippines.The author highly appreciates all the inputs from the conference session.

\section{REFERENCES}

Graizbord, D., Rodríguez-Muñiz, M., \& Baiocchi, G. (2017). Expert for a day: Theory and the tailored craft of ethnography. Ethnography, 18(3), 322-344.

Justice, A. (2015). Audio Visual Commission. Fontes Artis Musicae, 62(4), 319-321.

Matusiak, K. K., \& Johnston, T. K. (2012). Digitization as a Preservation Strategy Saving and Sharing the American Geographical Society Library's Historic Nitrate Negative Images. In proceedings of the International Conference, Memory of the World in the Digital Age: Digitization and Preservation, Vancouver, Canada. http://www.unesco.org/new/fileadmin/MULTIMEDIA/HQ/CI/CI/pdf/mow/VC_Matusiak_Johnston_28_B_1400.p df.

Müller, M. (2013). Challenges of Digitalization for the Music Industry. GRIN Verlag, https://www.grin.com/document/231543

Smith, J. A. (2015). Music Has Its Destiny: On Collecting Audio in a Digital Age. Audio Visual Commission Session, Sound Recording and Digital Libraries, June.

Warren, R., Maniscalco, M., Schroeder, E., Oliver, J., Huitt, S., Lambert, D., \& Frisch, M. (2013). Restoring the Human Voice to Oral History: The Audio-Video Barn Website. The Oral History Review, 40(1), 107-125. http://www.jstor.org/stable/43863461 